\title{
Management of Isolated Demyelinating Episodes by North American Neurologists
}

\author{
Alex Tselis, Seemant Chaturvedi, Mark Borsody, Rohan Vaidya
}

Can. J. Neurol. Sci. 2007; 34: 375-376

Previous studies have shown a disparity in the treatment practices of United States (US) and Canadian (C) neurologists in areas pertaining to stroke such as use of intravenous heparin for acute stroke and treatment recommendations for asymptomatic carotid stenosis. ${ }^{1,2}$ Whether US and Canadian neurologists differ in their evaluation and treatment of other neurologic conditions is unclear.

We undertook a survey to compare how US and Canadian neurologists evaluate clinically isolated syndromes (CIS) suggestive of demyelinating disease. We were specifically interested in the impact of the Controlled High Risk Avonex Multiple Sclerosis Prevention Study (CHAMPS) on neurologist treatment practices. ${ }^{3}$ Our hypothesis was that US neurologists would prescribe disease modifying drugs (DMDs) more frequently than Canadian neurologists.

\section{SubJects AND Methods}

A survey was taken of 140 neurologists from the United States and 140 neurologists from Canada. All were active members of the American Academy of Neurology. A systematic sample of names was chosen from the 2003-2004 American Academy of Neurology directory. The sampling methodology involved selecting the first and second name of the active members on each page of the directory for US neurologists and all of the active members for the $\mathrm{C}$ neurologists. We excluded pediatric neurologists and subspecialists who are unlikely to treat patients with CIS.

We distributed a questionnaire to the neurologists (Table), in which they were asked how they would evaluate and manage an instance of a patient with retrobulbar optic neuritis $(\mathrm{ON})$ and manage a patient with an internuclear ophthalmoplegia (INO) with two plaques on a brain MRI. The respondents were also asked about their familiarity with CHAMPS, with the degree that medicolegal considerations would influence their management decisions and how long they had been in practice. We also asked about their use of other DMDs used in MS: subcutaneous interferon $\beta-1 b\left(\right.$ Betaseron $\left.^{\mathrm{R}}\right)$, subcutaneous interferon $\beta-1 \mathrm{a}$ $\left(\right.$ Rebif ${ }^{\mathrm{R}}$ ), and subcutaneous glatiramer acetate $\left(\right.$ Copaxone ${ }^{\mathrm{R}}$ ). Responses of the US and Canadian neurologists were compared with chi square testing or Fisher's exact test.

Our institution does not require IRB approval for an anonymous survey. The work was supported by departmental funds.

\section{RESULTS}

The response rate was 37\%, with 47 US neurologists and 57 Canadian neurologists responding. There was no statistically significant difference in the response rate between US and Canadian neurologists $(\mathrm{p}=0.27)$. United States neurologists were more likely than Canadian neurologists to treat isolated optic neuritis with intravenous corticosteroids $(41 / 47,87 \%$ vs. $35 / 57$, $61 \%, \mathrm{p}=0.004)$, more likely to perform a lumbar puncture during the evaluation $(28 / 47,60 \%$ vs. $8 / 57,14 \%, \mathrm{p}<0.0001)$ and less likely to observe an $\mathrm{ON}$ patient with two periventricular MRI lesions and positive CSF oligoclonal bands (8/47, 17\% vs. $32 / 57,56 \%, \mathrm{p}<0.0001)$. Both groups would perform a visual evoked potential in about one half of the cases (US $55 \%$ vs. C $49 \%, \mathrm{p}=0.56$ ) and there was a trend for US neurologists to order a MRI of the brain more frequently (US $100 \%$ vs. C $91 \%$, $\mathrm{p}=0.06$ ). For a hypothetical patient with INO and two demyelinating plaques on MRI, US neurologists were again less likely to observe the patient, $(11 / 47,23 \%$ vs. $38 / 57,67 \%$, $\mathrm{p}<0.0001)$. Canadian neurologists were more likely to report familiarity with the results of CHAMPS, (50/55, 91\% vs. 35/47, $74 \%, \mathrm{p}=0.03)$. There were more US neurologists reporting medicolegal considerations as important in their management of CIS, $(6 / 46,13 \%$ vs. $3 / 57,5 \%)$, but this was not statistically significantly different $(\mathrm{p}=0.29)$.

For the ON scenario, more US neurologists were willing to prescribe an alternative DMD to intramuscular interferon $\beta$-1a $(37 / 47,79 \%$ vs. $6 / 57,11 \%, p<0.0001)$. For the INO scenario, the results were similar, $(33 / 47,70 \%$ vs. $4 / 57,7 \%, \mathrm{p}<0.0001)$.

\section{Discussion}

Our study shows significant differences between US and Canadian neurologists' approaches to the evaluation and management of patients with CIS. The US neurologists were more likely to use IV steroids for optic neuritis treatment,

From the Department of Neurology, Wayne State University School of Medicine, 8DUHC, Detroit, MI, USA.

ReCeived DeCEMber 13, 2006. AcCePted In FinAl fORM April 7, 2007 Reprint requests to: Alex Tselis, Department of Neurology, Wayne State University School of Medicine, 8D-UHC, 4201 St. Antoine, Detroit, MI 48201 USA. 


\section{Table: The Survey}

1. A 30-year-old woman comes to your office with unilateral visual loss and painful eye movement and you feel the diagnosis is retrobulbar optic neuritis. How would you treat her?(Observation, Oral prednisone, IV corticosteroids)

2. During evaluation for the optic neuritis, would you do the following tests: (VEP, lumbar puncture, brain MRI with contrast). If the CSF shows oligoclonal bands and if the brain MRI shows two periventricular white matter lesions, which of the following treatments would you prescribe: (Avonex, Betaseron, Copaxone, Rebif, None)

3. Which of the following treatments would you prescribe for a 40year-old woman with one episode of internuclear ophthalmoplegia, an otherwise normal exam, and a MRI scan with two demyelinating plaques? (Avonex, Betaseron, Copaxone, Rebif, None)

4. Are you familiar with the CHAMPS Study? (Yes, No)

5. Would you prescribe Avonex for patients with isolated demyelinating syndromes because you thought it was the medicolegal "standard of care"? (Yes, this would be a major factor; Yes, but this is not a major factor; No, continue to observe; No, but I would prescribe another drug: (circle which one) (Avonex, Betaseron, Copaxone, Rebif, None)

6. How long have you been in practice? $(<10$ years, $10-20$ years, $>20$ years)

perform a lumbar puncture during the diagnostic evaluation, and prescribe an immunomodulator besides intramuscular interferon $\beta$-1a for a CIS.

Canadian neurologists were more likely to observe the patient after an episode of ON or INO and were more familiar with the CHAMPS study. Neither group felt that medicolegal factors contributed to treatment decisions.

Our study reinforces the previous observations in stroke scenarios. In the use of intravenous heparin for acute stroke, US neurologists were more likely to use IV heparin despite the lack of clinical trial data supporting the use of heparin. ${ }^{1}$ The US neurologists were also more likely to refer a patient with asymptomatic carotid stenosis for carotid endarterectomy. ${ }^{2}$ In the current study, US neurologists were more likely to treat a CIS with IV steroids. These variations in treatment practices may reflect a "pressure to intervene" for US neurologists, which may at least be reflective of societal demands. It is also possible that Canadian neurologists receive more training in evidence-based medicine and apply the data in a more narrow fashion. Economic factors may also play a role since some Canadian provincial governments will only cover interventions such as intramuscular interferon $\beta-1 \mathrm{a}$, which have been subjected to clinical trial but not other DMDs which, at the time of the survey, had not been investigated in large trials. The tendency of US physicians to intervene more than their Canadian counterparts has also been shown in the treatment of coronary artery disease. ${ }^{4}$
The decreased availability of MRI scanning in Canada compared to the US has been well documented, although there has been a $>600 \%$ increase in the annual number of MRI scans in Ontario between 1993-2000. ${ }^{35}$ We found a trend for US neurologists to order MRI more frequently for patients with a CIS, which may partly reflect the easy availability of this imaging modality in the US.

Our study has limitations including a moderate response rate. In addition, we do not have detailed information regarding the practice settings (academic vs. community practice, volume of patients with demyelinating disease, etc.), practitioner subspecialties, regional variations within each country or patient volume of the respondents.

Despite these limitations, our study found significant practice differences and extends earlier observations on the variations in treatment approaches between US and Canadian neurologists. Additional studies examining the reasons for this "therapeutic divide" would be of interest.

\section{REFERENCES}

1. Al-Sadat A, Sunbulli M, Chaturvedi S. Use of intravenous heparin by North American neurologists: Do the data matter? Stroke. 2002; 33:1574-7.

2. Chaturvedi S, Meinke JL, St. Pierre E, Bertasio B. Attitudes of Canadian and U.S. neurologists regarding carotid endarterectomy for asymptomatic stenosis. Can J Neurol Sci. 2000; 27:116-9.

3. Jacobs LD, Beck RW, Simon JH, Kinkel RP, Brownscheidle CM, Murray TJ, et al. The Champs Study Group. Intramuscular interferon beta-1a therapy initiated during a first demyelinating event in multiple sclerosis. N Engl J Med. 2000; 343:898-904.

4. Tu JV, Pashos CL, Naylor CD, Chen E, Normand S-L, Newhouse $\mathrm{JP}$, et al. Use of cardiac procedures and outcomes in elderly patients with myocardial infarction in the United States and Canada. N Engl J Med. 1997; 336:1500-5.

5. Laupacis A, Evans W. Diagnostic imaging in Canada. Healthc Pap. $2005 ; 6: 8-15$ 